\title{
Compressão de sinais de eletrocardiogramas com wavelets determinadas por otimização genética
}

\author{
Rosanna M. R. Silveira, Cristiano M. Agulhari, Ivanil S. Bonatti e Pedro L. D. Peres
}

\begin{abstract}
Resumo - Um método de compressão baseado na determinação da wavelet que melhor se ajusta ao sinal de eletrocardiograma a ser comprimido é proposto neste artigo. Os coeficientes do filtro escala que minimizam a distorção do sinal comprimido são usados para determinar a wavelet. A escolha do filtro escala é feita pela parametrização dos coeficientes escala de modo que todas as restrições são satisfeitas para qualquer conjunto de parâmetros. Um algoritmo genético é utilizado para determinar os parâmetros que minimizam a distorção do sinal comprimido. O desempenho do algoritmo proposto é analisado e comparado com a compressão utilizando a wavelet clássica Db3.
\end{abstract}

Palavras-Chave - Compressão, Eletrocardiogramas, ECG, Wavelet, e Otimização genética.

Abstract-A compression method, based on the choice of a wavelet that matches the electrocardiogram signal to be compressed, is proposed in this paper. The coefficients of the scaling filter that minimize the distortion of the compressed signal are used to determine the wavelet. The choice of the scaling filter is done by the parameterization of the scaling coefficients in a way that all the constraints are satisfied for any set of parameters. A genetic algorithm is used to determine the parameters that minimize the distortion of the compressed signal. The performance of the proposed algorithm is analyzed and compared to the compression using the classical wavelet Db3.

Keywords - data compression, electrocardiogram signals, ECG, wavelet and genetic algorithm.

\section{INTRODUÇÃO}

A compressão de dados (com ou sem perdas) continua sendo uma componente importante nos sistemas de comunicações e armazenamento. Na compressão sem perdas os dados descomprimidos precisam ser idênticos aos dados originais. Tal característica é imprescindível, por exemplo, no armazenamento de textos cuja fidelidade precisa ser preservada [1]. Na compressão com perdas, o usuário pode controlar o compromisso entre alta qualidade e maior compressão adequando o algoritmo utilizado à aplicação em questão.

A maioria dos algoritmos de compressão com perdas pertence às seguintes categorias [2]:

- Métodos de compressão diretos, que detectam redundâncias pela análise das amostras do sinal;

Rosanna M. R. Silveira, Instituto Nacional de Telecomunicações, INATEL, Santa Rita do Sapucaí, MG, Brasil, E-mail: rosannas@inatel.br.

Cristiano M. Agulhari, Ivanil S. Bonatti e Pedro L. D. Peres, Departamento de Telemática, FEEC, UNICAMP, Campinas, SP, Brasil, E-mails: \{agulhari, ivanil, peres\}@dt.fee.unicamp.br.
- Métodos de reconhecimento de padrões, em que alguns parâmetros do sinal são extraídos e utilizados para compressão;

- Métodos preditivos, que quantizam e codificam o erro entre os dados originais e os dados preditos; ou

- Métodos de transformadas, que representam o sinal em um outro domínio apropriado para detecção e remoção de redundâncias.

$\mathrm{O}$ algoritmo de compressão baseado em wavelets proposto neste artigo pertence à última categoria. O objetivo da maioria das pesquisas sobre wavelets é criar um conjunto de funções base que resultam em descrições apropriadas do sinal em estudo. Na série de Fourier, senóides são escolhidas como funções base e as propriedades da expansão resultante são examinadas [3]. No caso da análise wavelet, as propriedades desejadas são definidas e então as funções base são determinadas. Uma wavelet é uma onda cuja energia é concentrada no tempo para prover uma ferramenta para a análise de fenômenos transitórios. Os sistemas wavelet são construídos a partir de uma ou mais funções escalas utilizando operações de escalonamento e translação. Praticamente todos os sistemas wavelet satisfazem as condições de multi-resolução, isto é, a expansão e contração dos sinais da base e os deslocamentos são feitos em múltiplos inteiros, tipicamente igual a dois. Os coeficientes de mais baixa resolução podem ser calculados a partir dos coeficientes de mais alta resolução através de um banco de filtros. Uma vez que existem diversas wavelets, elas podem ser escolhidas para se adaptar a aplicações individuais ajustando-se ao sinal sob estudo [4].

Técnicas de compressão utilizando wavelets têm sido aplicadas em diversos setores da engenharia com eficiência, especialmente em sinais de eletrocardiograma (ECG) [5]. Um ECG é um conjunto de sinais elétricos medidos por eletrodos colocados em contato com o corpo humano. Os ECGs são quase periódicos, com períodos de aproximadamente um segundo, e consistem de sinais elétricos associados aos batimentos cardíacos. Algumas doenças cardíacas podem ser diagnosticadas a partir de análises do sinal ECG feitas por profissionais especializados. A eficiência da compressão de sinais ECG utilizando wavelets depende da forma da wavelet, motivando diversas pesquisas na escolha de diferentes formas de onda para esta técnica [6], [7], [8], [9], [10], [11], [12], [13] e [14].

Alguns autores consideram a energia do sinal nos diferentes subespaços wavelet para selecionar a função wavelet mais apropriada para comprimir o sinal. Guido et al. [15] resolvem um sistema linear de equações composto 
pela derivada da energia de projeção no subespaço escala com relação a cada coeficiente escala e um conjunto de restrições que leva em conta os momentos da wavelet. O sistema é resolvido usando o método dos mínimos quadrados resultando em um filtro passa baixas que corresponde aos coeficientes escala. Gupta et al. [16] resolvem um sistema linear de equações composto pela derivada da energia do erro de projeção no subespaço wavelet com relação a todos os coeficientes escala exceto um, cujo valor é fixado em 1. Para simplificar o cálculo dos coeficientes, ambos os artigos consideram uma versão relaxada da maximização da energia de projeção no subespaço escala. No entanto, não podem garantir que a wavelet obtida maximiza a energia projetada.

O foco deste trabalho está na formulação da busca da função escala que resultaria na melhor compressão como um problema de minimização nos coeficientes escala. Os coeficientes escala são parametrizados [17] de forma que as restrições sobre os coeficientes sejam intrinsecamente satisfeitas e o problema de otimização seja reduzido a uma busca no espaço dos parâmetros. Um algoritmo genético é utilizado para encontrar o conjunto de parâmetros que resultaria na melhor compressão do sinal. O método consiste em encontrar a função escala mais apropriada a cada eletrocardiograma a ser comprimido, e os parâmetros que geram a função escala são armazenados junto com os dados comprimidos.

O restante do artigo é organizado conforme descrito a seguir. A seção II apresenta o método de compressão do sinal ECG. A seção III apresenta as propriedades principais das funções escala e wavelet do ponto de vista da análise multi-resolução e descreve um método para parametrizar os coeficientes escala. A seção IV apresenta o método proposto para escolha da wavelet que melhor se adapta ao sinal em estudo. A seção V apresenta alguns experimentos numéricos usando sinais ECG para avaliar o método proposto e a seção VI conclui o artigo.

\section{Compressẽo ECG}

O objetivo dos métodos de transformada, no contexto da compressão, é concentrar a energia do sinal em uma pequena quantidade de coeficientes transformados. Conseqüentemente é possível descartar os menores coeficientes sem aumentar consideravelmente a distorção do sinal reconstruído.

Em linhas gerais, o método de compressão de ECGs, no qual a escolha da wavelet será feita, consiste em:

- Pré-processamento dos sinais de ECG de longa duração realizando sua segmentação e introduzindo um certo número de zeros nas extremidades dos segmentos. Este procedimento é necessário se a wavelet utilizada der origem a significativa distorção nas bordas. O pré-processamento pode também ser utilizado para remover as componentes de baixa freqüência do sinal (como a média e a linha-base).

- Seleção dos coeficientes wavelet significativos pela retenção dos maiores coeficientes calculados pela decomposição wavelet do sinal de ECG. O número de coeficientes significativos é dado por

$$
N_{\eta}=\sigma N
$$

sendo $N_{\eta}$ o número de coeficientes significativos no nível $\eta$ de decomposição wavelet, $N$ o número de amostras do sinal e $\sigma$ a taxa de retenção previamente especificada. A taxa de compressão resultante e a distorção são altamente dependentes do número de coeficientes significativos e da wavelet escolhida. A principal contribuição deste artigo está no procedimento de determinação da wavelet.

- A codificação da posição dos coeficientes significativos, cuja posição é definida por uma cadeia de zeros e uns (se o coeficiente é significativo existe um correspondente 1 na cadeia, de outra forma um 0 é utilizado). A cadeia é codificada utilizando uma codificação de tamanho variável (variable run length code) [18] que, em geral, apresenta um bom desempenho, pois a cadeia pode conter seqüências longas de zeros.

- Codificação dos coeficientes significativos usando um código fonte como o código de Huffman [19] aplicado a uma versão quantizada dos coeficientes significativos. O número de níveis de quantização afeta tanto a distorção quanto a taxa de compressão.

Em linhas gerais, o procedimento de descompressão do sinal de ECG consiste em:

- Decodificação dos coeficientes significativos.

- Reconstrução das amostras do sinal de ECG pela execução do procedimento de reconstrução wavelet. As posições dos coeficientes não-significativos são preenchidas com zero.

- Obtenção do sinal reconstruído adicionando a média e a linha-base ao sinal.

A taxa de compressão (CR Compression Ratio) é definida como

$$
C R=\frac{s}{c+p+h}
$$

sendo $s$ o número total de bits das amostras originais do sinal de ECG, $c$ o número total de bits utilizados para codificar todos os coeficientes significativos, $p$ o número total de bits resultantes da codificação aplicada à cadeia com a posição dos coeficientes significativos e $h$ um cabeçalho contendo os parâmetros da fase de pré-processamento e os parâmetros que definem a wavelet escolhida.

A distorção (PRD Percent Root-mean-square Distortion) é dada por

$$
\mathrm{PRD}=\left(\frac{\sum_{k}(\nu[k]-\hat{\nu}[k])^{2}}{\sum_{k} \nu^{2}[k]}\right)^{1 / 2} \times 100
$$

sendo $\nu[k]$ o sinal original e $\hat{\nu}[k]$ o sinal reconstruído.

\section{FunÇÕES EscAla E Wavelet}

Uma função real não nula $\varphi(x)$ que pode ser expressa pela soma ponderada de deslocamentos da função con- 
traída $\varphi(2 x)$ como

$$
\varphi(x)=\sum_{k} \ell[k] \sqrt{2} \varphi(2 x-k), \quad k \in \mathbb{Z}, \quad x, \ell[k] \in \mathbb{R}
$$

é conhecida como função escala, sendo $\ell[n]$ os coeficientes escala. A Equação (4) é chamada de equação de multiresolução. A função escala pode ser complexa, mas neste artigo apenas funções reais são consideradas.

A wavelet $\psi\left(2^{\eta} x\right)$ é escrita como uma soma ponderada de deslocamentos da função escala $\varphi\left(2^{\eta+1} x\right)$ dada por

$$
\psi\left(2^{\eta} x\right)=\sum_{k} h[k] \sqrt{2^{\eta+1}} \varphi\left(2^{\eta+1} x-k\right),
$$

sendo $h[n]$ os coeficientes de representação da wavelet no subespaço $V_{\eta+1}$ [20], que tem como base o conjunto formado pelos deslocamentos inteiros da função $\varphi\left(2^{\eta+1}\right)$.

A existência de pares de funções $\varphi(x)$ e $\psi(x)$ satisfazendo ambas Equações (4) e (5) é a essência da decomposição wavelet que possibilita a técnica de compressão discutida neste artigo, sendo as funções Haar o primeiro par proposto na literatura [21].

Os coeficientes $h[n]$ são calculados como

$$
h[n]=(-1)^{n} \ell[m-n-1]
$$

para wavelets de suporte compacto, ou seja

$$
\begin{gathered}
\ell[n]=h[n]=0, n \notin\{0, \ldots, m-1\} \subset \mathbb{Z} \\
\varphi(x)=\psi(x)=0, x \notin[0, m-1] \subset \mathbb{R},
\end{gathered}
$$

sendo $m$ o número de coeficientes do filtro escala. Para wavelets ortonormais, $m$ é um número par [20].

\section{A. Projeção usando bancos de filtros}

A projeção ortogonal da função $f(x)$ no subespaço $V_{1}$ é dada por

$$
f_{1}(x)=\sum_{k} a_{1}[k] \varphi(2 x-k) .
$$

A função $f_{1}(x)$ pode também ser escrita como

$$
f_{1}(x)=\sum_{k} a_{0}[k] \varphi(x-k)+\sum_{k} b_{0}[k] \psi(x-k),
$$

sendo $a_{0}[n], b_{0}[n]$ os coeficientes de projeção da função $f(x)$ nos subespaços $V_{0}$ e $W_{0}$ (ortogonal e complementar a $\left.V_{0}\right)$, respectivamente.

O algoritmo de decomposição wavelet é dado por [4]

$$
a_{\eta-1}[n]=\left\{\ell[-n] * a_{\eta}[n]\right\}_{\downarrow 2}
$$

$\mathrm{e}$

$$
b_{\eta-1}[n]=\left\{h[-n] * a_{\eta}[n]\right\}_{\downarrow 2},
$$

sendo $*$ e $\{\cdot\}_{\downarrow 2}$ os operadores de convolução e downsample, respectivamente.

O algoritmo de reconstrução wavelet é dado por

$$
a_{\eta}[n]=\ell[n] *\left\{a_{\eta-1}[n]\right\}_{\uparrow 2}+h[n] *\left\{b_{\eta-1}[n]\right\}_{\uparrow 2}
$$

sendo $\{.\}_{\uparrow 2}$ o operador de upsample.

As amostras $f[n]$ da função contínua $f(x)$ podem ser vistas como os coeficientes de projeção do sinal $f(x)$ na base $\{\delta(x-k)\}$, ou como os coeficientes de projeção do sinal $f(x)$ na base $\{\operatorname{Sinc}(x-k)\}$ se a função e o intervalo entre as amostras satisfazem o teorema da amostragem de Shannon-Nyquist. As amostras $f[n]$ são os coeficientes de projeção do sinal $f(x)$ no subespaço $V_{\eta}$ se o número de amostras por unidade é $2^{\eta}$. Portanto, as Equações (10) e (11) permitem a obtenção dos coeficientes em uma taxa igual a $2^{\eta-1}$ através da aplicação das amostras $f[n]$ nos filtros $\ell[-n]$ e $h[-n]$.

O número $\kappa$ de amostras da versão discreta da função $\varphi(x)$ é dado por

$$
\kappa=(m-1) 2^{\eta-1}+1 .
$$

O número $M_{k}$ de coeficientes $b_{k}[n]$ é dado por

$$
M_{k-1}=\left\lfloor\frac{M_{k}+m-1}{2}\right\rfloor, \quad k=\{0, \ldots, \eta-1\}
$$

com $M_{\eta}=N$ (número de amostras de $f[n]$ ). Portanto, o número de coeficientes resultantes da decomposição é dado por

$$
T_{\eta}=M_{0}+\sum_{k=0}^{\eta-1} M_{k}
$$

\section{B. Parametrização}

As transformadas $L(z)$ da seqüência $\ell[n]$ e $H(z)$ da seqüência $h[n]$ formam um par de Quadrature Mirror Filters [17], [22], [23] e podem ser escritas como

$$
\left[\begin{array}{c}
L(z) \\
z^{-2(2 m-1)} H(z)
\end{array}\right]=E\left(z^{2}\right)\left[\begin{array}{c}
1 \\
z^{-1}
\end{array}\right],
$$

sendo a matriz de transferência $E(z)$ dada por

$$
E(z)=V_{\gamma}(z) V_{\gamma-1}(z) \cdots V_{1}(z) V_{0}
$$

com matrizes $V_{k}$ dadas por

$$
V_{k}(z)=\mathbf{I}+\left(z^{-1}-1\right) v_{k} v_{k}^{\prime}, \quad \text { para } 1 \leq k \leq \gamma-1
$$

e com vetores reais de norma unitária $v_{k}$.

Não há perda de generalidade se $v_{k}$ é escrito na forma

$$
v_{k}=\left[\begin{array}{c}
\cos \left(\theta_{k}\right) \\
\sin \left(\theta_{k}\right)
\end{array}\right]
$$

e a matriz $V_{0}$ na forma

$$
V_{0}=\frac{\sqrt{2}}{2}\left[\begin{array}{cc}
1 & 1 \\
1 & -1
\end{array}\right]
$$

O fator $z^{-2(2 m-1)}$ é usado para satisfazer a Equação (6) e o grau $\gamma$ da matriz $E(z)$ é igual ao número de atrasos $\left(z^{-1}\right)$ necessários para implementar os filtros. No contexto de parametrização das wavelets, $\gamma$ é igual ao grau de liberdade na escolha da função escala, isto é,

$$
\gamma=\frac{m}{2}-1
$$

Em suma, os passos para determinar os coeficientes escala $\ell[n]$ a partir dos parâmetros $\theta$ são:

- Calcule os polinômios $E(z)$ (Equação (17));

- Calcule $L(z)=E_{11}\left(z^{2}\right)+z^{-1} E_{12}\left(z^{2}\right)$ (Equação (16));

- Obtenha $\ell[n]$ da expressão

$$
L(z)=\ell[\gamma-1] z^{-(\gamma-1)}+\ldots+\ell[1] z^{-1}+\ell[0] .
$$


Distintas funções escala produzem diferentes coeficientes $a_{0}[n]$ e $b_{k}[n], k \in\{0, \ldots, \eta-1\}$.

Na próxima seção, um método para escolha da função escala $\varphi(x)$ que resulta em valores de pequena amplitude para os coeficientes $b_{k}[n]$ e valores de maior amplitude para os coeficientes $a_{0}[n]$ é proposto como uma técnica para produzir bons algoritmos de compressão. Na compressão com perda, primeiro os coeficientes são computados e então os de maior amplitude são retidos (os de menor amplitude são substituídos por zero) para recuperar o sinal original.

\section{EscolHA DA Wavelet}

Algoritmos genéticos são modelos computacionais que são aptos a encontrar boas soluções para problemas de otimização, e são recomendados para problemas nãoconvexos. Muitos termos técnicos do estudo de genética são incorporados na aplicação dos algoritmos genéticos.

Cada possível solução do problema de otimização é chamada de cromossomo ou um indivíduo, e um conjunto de indivíduos é chamado de população. O algoritmo se inicia com a geração aleatória de uma população inicial cujo tamanho é um número par tipicamente igual a 20. A nova população é gerada selecionando-se alguns indivíduos da população atual. Esta seleção é feita atribuindo-se um valor de aptidão para cada indivíduo (que é o valor da função objetivo quando aplica-se o valor associado ao indivíduo). Uma probabilidade de seleção é determinada para cada indivíduo baseada no valor de aptidão, logo os indivíduos que apresentam os melhores valores possuem maiores chances de compor a população na próxima geração. Note que um indivíduo pode ser escolhido várias vezes, bem como pode não ser escolhido. Então, a operação de crossover é aplicada combinando cada dois indivíduos para gerar novos dois. A operação de mutação pode também ser aplicada nos indivíduos restantes introduzindo pequenas mudanças em cada indivíduo. Estes procedimentos são repetidos até que a população convirja para um mínimo local da função.

O seguinte problema de otimização precisa ser resolvido a fim de encontrar a wavelet que resulta em um mínimo local da distorção do sinal de ECG considerado para uma dada taxa $\sigma$ de retenção (Equação(1)):

$$
\min _{\theta} \operatorname{PRD}(\theta) \text {. }
$$

O algoritmo genético aplicado à Equação (22) foi implementado usando o pacote Genetic Algorithm Tool do Matlab, versão 7.0.1.24704 (R14).

$\mathrm{O}$ algoritmo genético considera cada conjunto $\theta$ como um indivíduo sendo $\operatorname{PRD}(\theta)$ calculado pelos seguintes passos:

1) Dado um conjunto de parâmetros $\theta_{k} \in[0, \pi], k \in$ $\{1,2, \ldots, \gamma\}$, encontre os coeficientes escala e wavelet conforme descrito na seção III-B;

2) Calcule a resolução $\eta$ por

$$
\eta \approx \log _{2}\left(\frac{2(\rho-1)}{m-1}\right)
$$

sendo $\rho$ o número de amostras de um batimento do sinal de ECG. O valor de $\eta$ é computado para produzir $\kappa$ (número de amostras da função escala) dado pela Equação (13) aproximadamente igual a $\rho$;

3) Decomponha o sinal de ECG na resolução $\eta$ conforme descrito na Seção III-A, mantendo os $N_{\eta}$ maiores coeficientes e substituindo os coeficientes restantes por zero, onde $N_{\eta}$ é dado pela Equação (1) para uma taxa de retenção $\sigma$ previamente definida;

4) Calcule o sinal reconstruído $\hat{\nu}_{\theta}[n]$ usando a Equação (12) e a distorção quadrática $\operatorname{PRD}(\theta)$ usando a Equação (3).

Note que o sinal reconstruído $\hat{\nu}_{\theta}[n]$ depende do conjunto de parâmetros $\theta=\left\{\theta_{1}, \theta_{2}, \ldots, \theta_{\gamma}\right\}$; tal conjunto define os coeficientes $\ell[n]$ e $h[n]$, que definem as funções escala e wavelet (base para o sinal $\hat{\nu}_{\theta}[n]$ ).

\section{Experimentos NumÉRICOS}

O objetivo dos experimentos é comparar a distorção quando utilizada a wavelet resultante do método proposto com a distorção produzida por uma wavelet clássica [24]. A taxa de retenção é $\sigma=4$, o número de coeficientes do filtro escala é $m=10$ e o nível de decomposição é $\eta=6$.

Os sinais de ECG utilizados nos experimentos pertencem ao banco de dados de sinais de eletrocardiogramas do "Research Resource for Complex Physiologic Signals" [25]. O acesso aos bancos de dados do PhysioBank [26] e os algoritmos são implementados utilizando Matlab. PhysioBank é um grande e crescente arquivo de gravações digitais de sinais fisiológicos e dados relacionados para a comunidade de pesquisa biomédica. Atualmente contém bancos de dados de sinais cardiopulmonares multiparâmetros, neurais e outros sinais biomédicos advindos de pacientes saudáveis e também de pacientes apresentando uma variedade de características como ataque cardíaco, epilepsia, apnéia e envelhecimento.

O banco de dados para testes de compressão do MITBIH [27] contém 168 gravações de sinais de ECG (com 20.48 segundos cada um, correspondendo a 5120 amostras) selecionados para testar uma variedade de desafios aos métodos de compressão. Estes 168 sinais foram separados em 38 grupos e um sinal foi extraído de cada grupo para que fossem realizados testes no método de compressão proposto.

Os valores dos parâmetros resultantes do método proposto para os sinais 08730_01_0 e 13030_03_0 [26] são apresentados na Tabela I. Os valores de PRD obtidos usando o método proposto e usando a wavelet clássica Db3 são mostrados na Tabela II.

TABELA I

VALORES DOS PARÂMETROS DA FUNÇÃo ESCALA OBTIDOS PELO MÉTODO PROPOSTO.

\begin{tabular}{|c|c|c|c|c|}
\hline Sinal & $\theta_{1}$ & $\theta_{2}$ & $\theta_{3}$ & $\theta_{4}$ \\
\hline 08730_01_0 & 0.5349 & 1.8898 & 1.3962 & 1.6535 \\
13030_03_0 & 0.3934 & -0.1258 & -2.5924 & 2.0877 \\
\hline
\end{tabular}


TABELA II

VALORES DE PRD USANDO A wavelet RESUlTANTE DO MÉTODO PRoposto (COLUNA "PRD-MÉTODO") E USANDO A wavelet CLÁSSICA Dв3.

\begin{tabular}{|c|c|c|}
\hline Sinal & PRD-método & PRD-Db3 \\
\hline 08730_01_0 & 3.69 & 3.82 \\
13030_03_0 & 2.16 & 2.27 \\
\hline
\end{tabular}

A wavelet Db3 foi escolhida para ser comparada ao método proposto uma vez que é apontada como a melhor wavelet para comprimir sinais de ECG de acordo com [24]. Note que os resultados obtidos pelo método proposto são melhores que aqueles obtidos usando a wavelet clássica (valores menores de PRD).

As Figuras 1 e 2 mostram um batimento de dois sinais de ECG e as funções escala correspondentes obtidas pelo método proposto. Note que o formato das funções escala é similar ao formato dos respectivos batimentos.

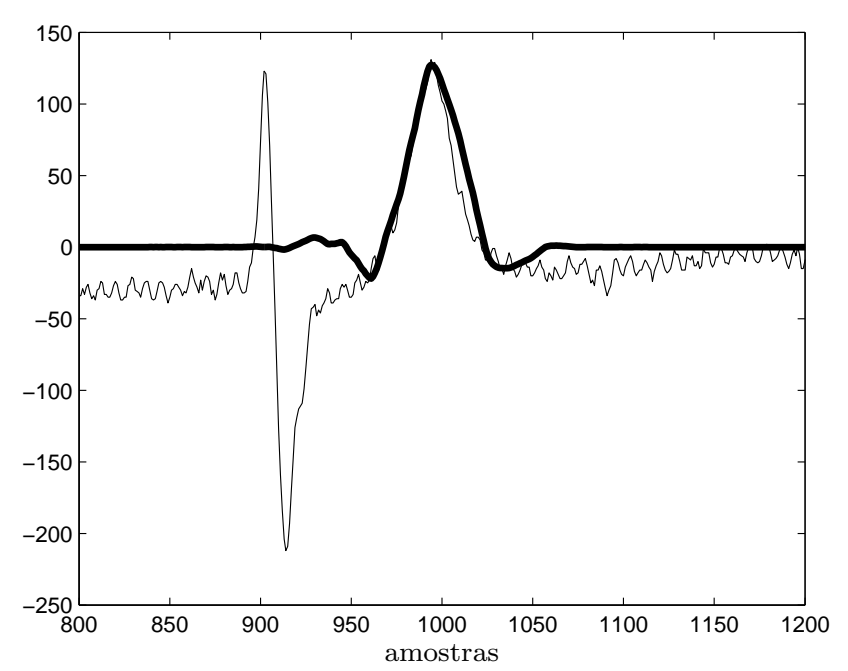

Fig. 1. Um batimento do sinal de ECG 08730_01_0 (linha fina) e a função escala correspondente.

Os valores de distorção (PRD) obtidos usando o método proposto e usando a wavelet Db3, para 38 sinais de ECG, são mostrados na Figura 3. Cada ponto na figura é associado a um sinal particular de ECG, sendo a coordenada $\mathrm{X}$ referente ao PRD do método proposto e a coordenada $\mathrm{Y}$ referente ao PRD produzido pela wavelet Db3. Note que o método proposto resulta em distorções menores que aquelas resultantes da wavelet $\mathrm{Db} 3$.

\section{CONClusões}

Uma nova técnica para encontrar uma wavelet que combina com um dado sinal foi proposto neste artigo. Tal técnica consiste na minimização da distorção do sinal comprimido usando um algoritmo genético na busca dos coeficientes escala parametrizados. Esta estratégia tem a vantagem de que as principais restrições nos coeficientes escala estão embutidas na parametrização, facilitando a

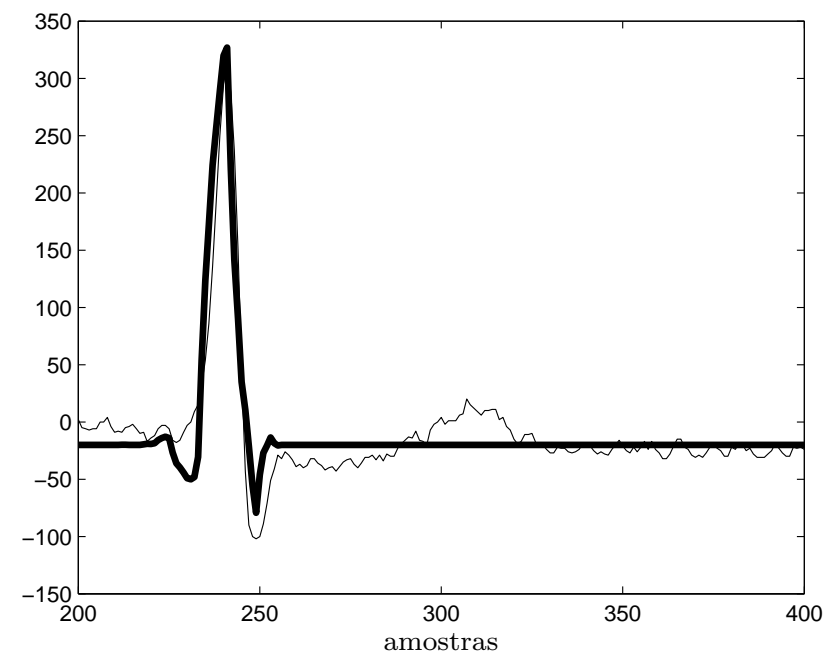

Fig. 2. Um batimento do sinal de ECG 13030_03_0 (linha fina) e a função escala correspondente.

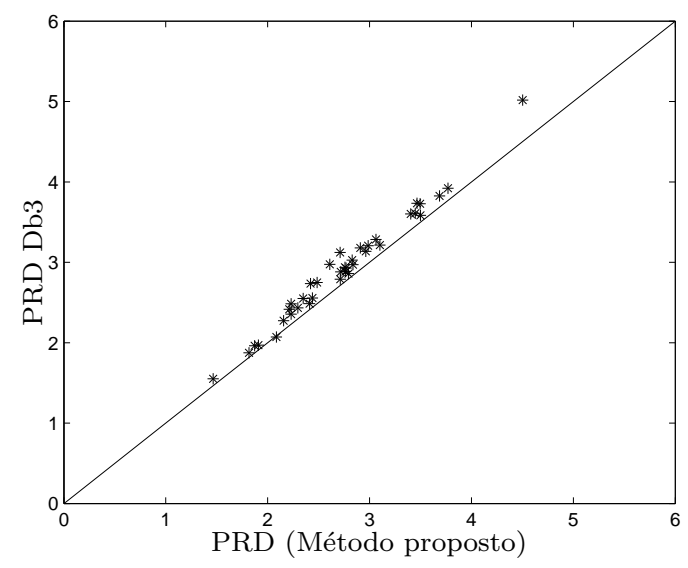

Fig. 3. Valores de PRD obtidos utilizando o Db3 e a wavelet obtida pelo método proposto aplicados a 38 sinais de ECG.

busca por wavelets adequadas, e o algoritmo genético se mostrou apto para lidar com os diversos mínimos locais das funções de distorção não-convexas. Experimentos numéricos foram conduzidos a fim de comparar a compressão utilizando o método proposto com a compressão usando a wavelet clássica Db3 e mostraram que o método proposto neste artigo apresenta melhores resultados.

\section{Agradecimentos}

Este trabalho foi apoiado por CNPq, CAPES, FAPESP e INATEL.

\section{REFERÊNCIAS}

[1] J. A. Storer, Data compression: methods and theory. New York, NY, USA: Computer Science Press, Inc., 1988.

[2] S. M. S. Jalaleddine, C. G. Hutchens, R. D. Strattan, and W. A. Coberly, "ECG data compression techniques - a unified approach," IEEE Transactions on Biomedical Engineering, vol. 37, pp. 329 - 343, April 1990. 
[3] A. V. Oppenheim and R. Schafer, Discrete-Time Signal Processing, 2nd ed., ser. Prentice Hall Signal Processing Series. Prentice Hall, 1999.

[4] C. S. Burrus, R. A. Gopinath, and H. Guo, Introduction to Wavelets and Wavelet Transforms - A Primer, 1st ed. Prentice Hall, 1998

[5] M. L. Hilton, "Wavelet and wavelet packet compression of electrocardiograms," IEEE Transactions on Biomedical Engineering, vol. 44, no. 5, pp. 394 - 402, May 1997.

[6] R. Benzid, F. Marir, A. Boussaad, M. Benyoucef, and D. Arar, "Fixed percentage of wavelet coefficients to be zeroed for ECG compression," Electronics Letters, vol. 39, no. 11, pp. 830 - 831, May 2003.

[7] B. Bradie, "Wavelet packet-based compression of single lead ECG," IEEE Transactions on Biomedical Engineering, vol. 43, no. 5, pp. 493 - 501, May 1996.

[8] J. Chen and S. Itoh, "A wavelet transform-based ECG compression method guaranteeing desired signal quality," IEEE Transactions on Biomedical Engineering, vol. 45, no. 12, pp. 1414 - 1419, Dec. 1998.

[9] R. S. H. Istepanian, L. J. Hadjileontiadis, and S. M. Panas, "ECG data compression using wavelets and higher order statistics methods," IEEE Transactions on Information Technology in Biomedicine, vol. 5, no. 2, pp. 108 - 115, June 2001.

[10] R. S. H. Istepanian and A. A. Petrosian, "Optimal zonal wavelet-based ECG data compression for a mobile telecardiology system," IEEE Transactions on Information Technology in Biomedicine, vol. 4, no. 3, pp. $200-211$, Sep. 2000.

[11] B. A. Rajoub, "An efficient coding algorithm for the compression of ECG signals using the wavelet transform," IEEE Transactions on Biomedical Engineering, vol. 49, no. 4, pp. 355 - 362, April 2002 .

[12] A. G. Ramakrishnan and S. Saha, "ECG coding by waveletbased linear prediction," IEEE Transactions on Biomedical Engineering, vol. 44, no. 12, pp. 1253 - 1261, Dec. 1997.

[13] S. G. Miaou, H. L. Yen, and C. L. Lin, "Wavelet-based ECG compression using dynamic vector quantization with tree codevectors in single codebook," IEEE Transactions on Biomedical Engineering, vol. 49, no. 7, pp. $671-680$, July 2002.

[14] Z. Lu, D. Y. Kim, and W. A. Pearlman, "Wavelet compression of ECG signals by the set partitioning in hierarchical trees algorithm," IEEE Transactions on Biomedical Engineering, vol. 47, no. 7 , pp. $849-856$, July 2000 .

[15] R. Guido, J. Slaets, R. Köberle, L. Almeida, and J. Pereira, "A new technique to construct a wavelet transform matching a specified signal with applications to digital, real time, spike, and overlap pattern recognition," Digital Signal Processing Elsevier, vol. 16, pp. 24-44, Jan. 2006.

[16] A. Gupta, S. D. Joshi, and S. Prasad, "On a new approach for estimating wavelet matched to signal," in 8th National Conference on Communications. IIT, 2002, pp. 180-184.

[17] H. Zou and A. H. Tewfik, "Parametrization of compactly supported orthonormal wavelets," IEEE Transactions on Signal Processing, vol. 41, no. 3, pp. 1428 - 1431, Mar. 1993.

[18] S. Golomb, "Run-length encoding," IEEE Transactions on Information Theory, vol. IT-12, pp. 399 - 401, 1966.

[19] R. B. Ash, Information Theory. Dover Publications, Inc., 1965.

[20] I. Daubechies, Ten Lectures on Wavelets, 8th ed. Society for Industrial and Applied Mathematics, 1992.

[21] G. Strang, Signal processing for everyone, ser. Computational Mathematics Driven by Industrial Problems. Springer Lecture Notes in Mathematics, 1999.

[22] P. P. Vaidyanathan, "Multirate digital filters, filter banks, polyphase networks, and applications: A tutorial," Proceedings of the IEEE, vol. 78, no. 1, pp. 56 - 93, January 1990.

[23] G. Strang and T. Nguyen, Wavelets and Filter Banks. Wellesley - Cambridge Press, 1997.

[24] R. Besar, C. Eswaran, S. Sarib, and R. J. Simpson, "On the choice of the wavelets for ECG data compression," in Proceedings of 2000 International Conference on Acoustics, Speech, and Signal Processing ICASSP '00, vol. 6, June 2000, pp. 3614 3617 .

[25] A. L. Goldberger, L. A. N. Amaral, L. Glass, J. M. Hausdorff, P. C. Ivanov, R. G. Mark, J. E. Mietus, G. B. Moody, C.K. Peng, and H. E. Stanley, "PhysioBank, PhysioToolkit, and PhysioNet: Components of a new research resource for complex physiologic signals," Circulation, vol. 101, no. 23, pp. e215-e220, Jun. 2000.

[26] PhysioBank - Physiologic signal archives for biomedical research, available at http://physionet.incor.usp.br/physiobank.

27] G. B. Moody, R. G. Mark, and A. L. Goldberger, "Evaluation of the TRIM ECG data compressor," Computers in Cardiology, vol. 15 , pp. $167-170,1988$. 